\title{
Reaching measures of monocular distance perception: Forward versus side-to-side head movements and haptic feedback
}

\author{
EMILY A. WICKELGREN, DANIEL S. MCCONNELL, and GEOFFREY P. BINGHAM \\ Indiana University, Bloomington, Indiana
}

\begin{abstract}
We investigated whether forward or side-to-side head movements yielded more accurate and precise monocular egocentric distance information, as shown by performance in a reaching task. Observers wore a head-mounted camera and display to isolate the optic flow generated by their head movements and had to reach to align a stylus directly under a target surface. Performance in the two head movement conditions was also tested with normal monocular vision. We tested performance in the two head movement conditions when the observers were given haptic feedback and compared performance when haptic feedback was removed. Performance was both more accurate and more precise in the forward head movement condition than in the side-to-side head movement condition. Performance in the side-to-side condition also deteriorated more after the removal of haptic feedback than did performance in the forward head movement condition. In the normal monocular condition, performance was comparable for the two head movement conditions. The implications for enucleated patients are discussed.
\end{abstract}

A problem in perceiving definite distances is that spatial metrics are lost in the projection from surfaces into optical patterns (Bingham, 1993b; Bingham \& Pagano, 1998) ${ }^{1}$. So, how do people obtain information about definite distances? This problem is especially salient for enucleated patients (people who have had one eye removed and are thus permanently monocular), because they cannot use binocular vision to obtain distance information. Despite this, monocular people do not appear to have large problems performing everyday tasks that require perceiving the distance of objects. We do not normally see people walk into walls or misguide their reach when they aim to grab a coffee mug. So, definite distance must somehow be perceived. One possible source of information arises from the patterns of optic flow produced by voluntary self-movement, which is necessarily accompanied by somatosensory information about head movement. Such information about the distance or velocity of head movements could be used to scale optic flow information about distance (Bingham \& Stassen, 1994; S. Rogers \& B. J. Rogers, 1992).

The possibility of using head movements to scale distance is especially important for monocular observers. Servos, Goodale, and Jakobson (1992) compared the abilities of monocular and binocular observers to use vision to guide reaching. They found that in normal lighting, monocular observers underestimated distance, relative to binoc-

This research was supported by the National Institute of Health, NEI Grant 1 R01 EY11741-01A. We thank Michael Muchisky for assistance in data collection, as well as G. John Andersen and two anonymous reviewers for their helpful comments and suggestions on an earlier draft of this manuscript. Correspondence concerning this article should be addressed to E. A. Wickelgren, Department of Psychology, Indiana University, Bloomington, IN 47405 (e-mail: ewickelg@indiana.edu). ular observers. The monocular reaches took longer and had lower peak velocities, longer deceleration times, and smaller grip apertures (suggesting that the observers also underestimated the size of the objects). The reaches were performed without any prior deliberate head movements. Marotta, Perrot, Nicolle, Servos, and Goodale (1995) found that monocular observers spontaneously learned to move their heads to obtain distance information by which to guide reaches. However, no clear preference for a given direction of head movement was apparent. A cross-sectional study with enucleated people revealed that the longer the time after enucleation, the greater the amount of head nodding and head shaking (up/down and back and forth, respectively) and the smaller the amount of forward head movement (Marotta, Perrot, Nicolle, \& Goodale, 1995). No studies up to this point, however, have systematically investigated the differences in distance estimations when using forward versus side-to-side head movements.

A number of studies have been performed to investigate distance perception via absolute motion parallax generated by side-to-side head movements (Eriksson, 1974; Ferris, 1972; Foley, 1977, 1978; Foley \& Held, 1972; Gogel \& Tietz, 1973, 1979; Johansson, 1973; B. J. Rogers, 1993), but few have included measures of definite distance perception (see Bingham \& Pagano, 1998, for a discussion). Gogel and Tietz (1979) investigated how well monocular observers could perceive the distance of a single lighted point in the dark with side-to-side head movements. When mean judgments were plotted as a function of actual distances, the slope was less than one $(\approx .7)$, and nearer targets $(30 \mathrm{~cm})$ were overestimated, whereas far targets $(96.4 \mathrm{~cm})$ were judged accurately.

Bingham and Stassen (1994) showed that radial optic expansion generated by forward head movement contains 
information about distance. Bingham and Pagano (1998) found that observers could use information generated by forward head movements to guide reaches. In this case, seated observers performed reaches in order to place a stylus in a target at different distances within reach. The observers viewed the targets monocularly via a headmounted video monitor and camera (called the headcam), which isolated optic flow. This visual information was coupled with somatosensory information about the head motion used to generate the optic flow. Observers were also tested when using normal monocular and binocular vision. Bingham and Pagano found that observers using monocular vision with or without the headcam underestimated distances more as the actual distance of the target increased. Reaches performed using binocular vision were accurate. Although the use of the headcam isolated optic flow as information for distance, the authors did not investigate whether observers could use motion parallax to obtain distance.

Additional support for the use of information generated by forward head movement was provided in a study by Eriksson (1974), who had monocular observers make verbal judgments of the depths of three objects prior to and after walking directly toward and away from the objects. Prior to walking, the observers' judgments were inaccurate (the higher objects was usually seen to be farther away from the lower objects). Once the observers were allowed to generate optic flow information from walking forward, distance judgments were very close to the actual physical distance of the objects. In an animal study, Ellard, Goodale, and Timney (1984) found that monocular gerbils trained to jump over a gap employed larger up-and-down head movements than did binocular gerbils. However, when the monocular gerbils were allowed to approach the gap so as to generate optic flow from forward head motion, they did not make the up-and-down head movements, yet they jumped with equivalent accuracy.

The question remains which direction of head movement might best enable observers to perceive egocentric distance, especially when the information is to be used to guide an action such as reaching. Forward head movements performed by seated observers (as in the Bingham and Pagano, 1998, study) generate substantial amounts of both radial expansion and parallax in optical flow. This is because the head moves both forward and downward owing to rotation about the base of the neck or the trunk. Although some radial expansion is also generated by sideto-side head movements, the amount is relatively insignificant. Thus, one might expect forward head movements to be more efficient. Forward head movements are also more natural in the context of reaching, because one tends to move the trunk toward an object when reaching for it.

A direct comparison cannot be made with the results of previous studies, because of differences in response measures. Studies of motion parallax have used verbal estimates as a response measure. Pagano and Bingham (1998) compared verbal estimates and reaches and found that the errors in the two cases were uncorrelated. In addition, they found, as had Foley (Foley \& Held, 1972), that verbal per- formance was at least twice as variable as manual performance. Accordingly, Pagano and Bingham suggested that reaching must be used as a measure by which to evaluate the effectiveness of distance perception when used to guide reaching. Bingham and Pagano (1998) used reaching as a measure for definite distance perception but only had observers make forward head movements. In our experiment, we compare forward and side-to-side head movements, to see whether there is a difference in performance.

In the context of reaching, two different types of somatosensory information can be used to scale optical information about distance. In addition to kinesthetic and vestibular information about head movement, haptic feedback from contact with targets is usually available in each successive reach. Haptic feedback was available but was not manipulated in the Bingham and Pagano (1998) studies, making it impossible to assess how well people do with just the somatosensory information from head movements. In the following experiments, we test the importance of the haptic feedback by removing it and, hence, also test the effectiveness and stability of the somatosensory information from voluntary head movements. Bingham and Zaal (1997a, 1997b) have found that targeted reaches performed without such haptic feedback are unstable and eventually inaccurate as a result, with both dynamic binocular and monocular vision. Bingham and Zaal (1997a, 1997b) have also found that making haptic feedback available stabilizes the reaches and yields greater accuracy.

\section{EXPERIMENT 1}

In the present study, we investigated two related questions. First, which type of head movement, forward and back or side-to-side, generates optic flow allowing the most accurate and precise performance in targeted reaching? Second, how important is haptic feedback from contact with targets for accurate and precise performance?

Observers viewed targets at various distances via the headcam. Bright target disks were viewed monocularly in dark surrounds so that optic flow generated by head movement was isolated as information about distance.

\section{Method}

Participants. Five observers ( 3 male and 2 female) associated with Indiana University participated in this study. All 5 observers were right-handed. All had normal or corrected-to-normal vision. Two of the observers were the first two authors, and the remaining 3 were students in the psychology department, who were paid $\$ 5$ per hour for their participation and were naive about the experimental questions.

Apparatus. The observers were seated and reached to position a cylindrical plastic stylus, under a target disk. The observer held the stylus firmly in the right hand, so that $4.0 \mathrm{~cm}$ extended beyond the closed fist. The stylus was $18.5 \mathrm{~cm}$ in length, $1.0 \mathrm{~cm}$ in diameter, and weighed $23.2 \mathrm{~g}$. The Cartesian coordinates of infrared emitting diodes (IREDs) were measured, using a two-camera WATSMART kinematic measurement system (Northern Digital, Waterloo, Ontario, Canada). Positions were sampled at $100 \mathrm{~Hz}$ with a resolution of $\pm 3 \mathrm{~mm}$ and stored on a computer hard drive. Three IRED's were placed on a helmet, one IRED on the tip of the stylus, and another on one side of the target. Each trial began with the back end of the 
stylus inserted in a launch platform, which was located next to the observer's right hip. The stylus interrupted a beam in the launch platform. Recording was initiated when the stylus was removed. A WATSCOPE connected to the WATSMART recorded the signals from the launch platform.

A patch was placed over the observer's left eye. The observers wore a helmet, on which a miniature video camera and monitor were mounted. An eyepiece attached to the helmet and positioned over the right eye allowed the observers to view a monochrome video display. A camera lens (the headcam) was attached to the right side of the helmet, $9.0 \mathrm{~cm}$ to the right of the right eye, pointing forward and aligned with the sagittal and transverse planes of the head. The total weight of the helmet with viewer, lens, IREDs, and supporting hardware was $1.8 \mathrm{~kg}$. Control switches allowed the experimenter to control when the head-mounted display was switched on or off. The display was turned on manually by the experimenter at the beginning of each trial and was automatically switched off (with a delay of less than $10 \mathrm{msec}$ ) when the stylus was removed from the launch platform at the initiation of a reach.

The targets consisted of 18 flat round disks covered with smooth, textureless, white retroreflective tape. They were oriented perpendicular to the observer, so that they projected circular images. The targets were constructed of Plexiglas with great care so that there would be no features that would allow a given target to be distinguished and, thus, potentially used to obtain distance. We also decoupled retinal image size from distance, so that the observer could not identify the distance from a given image size. To do this, we used five different target sizes $(5,7,9,12$, and $15 \mathrm{~cm}$ in diameter) and constructed three identical targets of each size (to reduce the possibility that a particular target would be recognized and used to scale distance.). Since the targets were symmetric with respect to the vertical axis, each individual target could be placed at two orientations to the vertical (one side up or turned over with the opposite side up). Therefore, effectively, six targets could be used to produce a given retinal image size at a given distance (three targets of a given size with two orientations each). In addition, each target was used at more than one of the five target distances. Target distances were computed as a proportion of the observer's maximum reach. The five target distances were $.50, .58, .66, .76$, and .86 of the maximum reach. Two target sizes were used at two distances (the $5-\mathrm{cm}$ target at distances .5 and .58 , and the $15-\mathrm{cm}$ target at distances .76 and .86), whereas the other three target sizes were used at three distances each $(7 \mathrm{~cm}$ at $.5, .58$, and $.66 ; 9 \mathrm{~cm}$ at $.58, .66$, and $.76 ; 12 \mathrm{~cm}$ at $.66, .76$, and .86). Altogether, 78 different target configurations were used ( 2 target distances $\times 2$ target sizes $\times 3$ targets $\times 2$ orientations +3 target distances $\times 3$ target sizes $\times 3$ targets $\times 2$ orientations ). The extreme number of target configurations was created both so that retinal image size did not systematically vary with distance and so that a particular target could not be identified and used to judge distance (see Bingham \& Pagano, 1998, for the mean retinal image sizes used at each of the five distances). There was no correlation between retinal image size and distance $\left(r^{2}<.01\right)$, so retinal image size could not be used by the observers to predict distance. Retinal image size was not an experimental factor here; we only wanted to make sure it could not be a confounding variable.

The targets were illuminated by a fluorescent light with a parabolic reflector mounted above and behind the observer's head. When brightly illuminated, the target appeared in the head-mounted display as an isolated shape in a dark field. The brightness and contrast of the head-mounted display were adjusted to produce patch light images (Runeson \& Frykholm, 1981). The field was dark and structureless. The visible structure of the target was devoid of internal texture. Before each trial, one target from the set was placed at eye level at a given distance along a line extending from the camera lens, parallel to the sagittal plane of the observer. Target position was controlled by using mounts attached to an optical bench. To mask the sound of the target being positioned by the experimenter, the observer wore earphones, through which music was played between trials. Prior to starting the experiment, the observer adjusted the volume of the music to a comfortable level that masked the noise of the target positioning. The positioning apparatus was calibrated to the observer's eye position before each experimental session.

The target IRED was located $12 \mathrm{~cm}$ below and $3 \mathrm{~cm}$ to the right of the center of the target disk. The target position was measured by sampling the target IRED during the first 50 frames immediately after reach initiation. The coordinates were subsequently translated from the position of the IRED to the center of the target disk. The appropriate transformation was determined by measuring an IRED placed at the center of the target.

To obtain the reach distance of the observer, we omitted the last 25 frames of the stylus diode (the last $250 \mathrm{msec}$ ) and then averaged over the 25 preceding frames. We obtained mean $(x-, y-, z-)$ coordinates and took the largest of the corresponding standard deviations as an indicator of any extraneous hand movements or IRED reflection that occurred while the hand was held under the target. Either of these would cause an inaccurate measurement of reach distance. No extraordinarily large standard deviations were found, however, so no trials were omitted from the analysis as a result.

Procedure. The observers were instructed to move their heads either forward and back or side to side through four complete oscillations while observing the target. Note that the side-to-side movement is not a rotation of the head. The observers moved at the hip while holding the head level and oriented straight ahead and looking at the target. The observers then reached to place the stylus tip (at shoulder level) directly under the target disk (at eye level) without touching it. Initiation of the reach caused the display to be switched off. Thus, reaches were performed blindly. Once the observer had positioned the stylus under the target, he or she signaled the experimenter by saying "O.K." The experimenter then terminated WATSMART recording. Each observer's reaching performance was tested under four conditions: forward head movement with feedback, side-to-side head movement with feedback, forward head movement without feedback, side-to-side head movement without feedback. Feedback conditions were always performed before conditions without feedback. The order in which the observers experienced the head movement conditions was counterbalanced across the 5 observers. In the feedback conditions, the feedback was provided after the reach position had been recorded. The observer was allowed to move the stylus upward to place it in a plastic bottle cap $(2 \mathrm{~cm}$ in diameter) that had been affixed directly below the target disk. If the observer could not then find the bottle cap after an incorrect reach (since the hand would not be positioned at the correct distance directly below the cap), the experimenter directed the observer's hand to the bottle cap. The observers were not limited in the amount of time spent feeling the correct distance of the target (via the bottle cap). The observers were not permitted to view the hand relative to the target, so haptic contact was the only source of feedback. The no-feedback conditions (both forward and side to side) were run on the day following the feedback conditions. Each experimental session consisted of 25 experimental trials ( 5 target distances $\times 5$ target sizes) preceded by 12 practice trials. Therefore, the total number of trials run across the entire 2-day experiment was 148.

In all the conditions, the camera was turned off, and the headphones were turned on between trials, while the experimenter adjusted the size and distance of the target. The five target distances were presented in random order. A different random ordering of targets and distances was used in each condition for each observer. Several days before the experiment, each observer sat in the apparatus, with his or her back against the chair, and his or her maximum reach distance was measured. These distances were 82.5, 86.1, 79.3, 79.3, and $89.2 \mathrm{~cm}$ for Observers $1-5$, respectively. As was mentioned previously, the target distances presented to the observer during the experiment were determined as a proportion of the maximum reach distance. The five target distances were $.50, .58, .66, .76$, and .86 of the observer's maximum reach. 


\section{Results}

The results for each observer in each head movement and feedback condition are shown in Figure 1, where mean reach distances (with standard error bars) are plotted against target distances. The results are presented and analyzed in units proportional to maximum reach distances so that comparisons can be made across observers. First, we performed a multiple regression in which we regressed seven vectors on reach distances (Pedhazur, 1982). The first vector contained actual target distances. The second vector coded type of head movement (as $+1 /-1$ ). The third vector coded presence of feedback (as $+1 /-1$ ). These two vectors (second and third) tested the intercept difference in the relation between actual and reach distances. The fourth and fifth vectors tested the interactions or possible slope differences in the head movement and feedback conditions, respectively. The sixth vector tested the head movement $\times$ feedback interaction, and the final vector tested the three-way interaction. Nonsignificant factors were eliminated from the multiple regression until only significant factors remained (Pedhazur, 1982).

After removing two nonsignificant factors, the overall multiple regression was significant $\left[r^{2}=.46, F(5,485)=\right.$ $80.9, p<.001]$. There were significant main effects for both the head movement and the feedback conditions. Although performance in the side-to-side condition resulted in a significantly higher mean intercept (.26) than in the forward condition (.13; partial $F=7.26, p<.01$ ), this difference was accompanied by a nonsignificant trend for difference in slopes (partial $F=3.62, p=.06$ ). The side-

\section{Forward With Feedback}

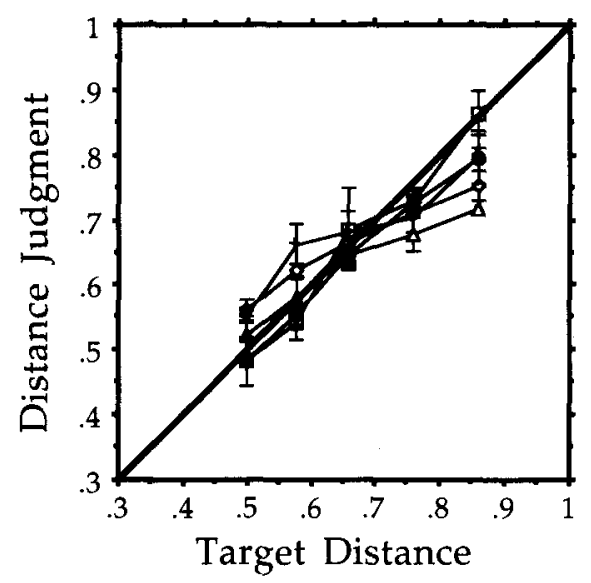

Side to Side With Feedback

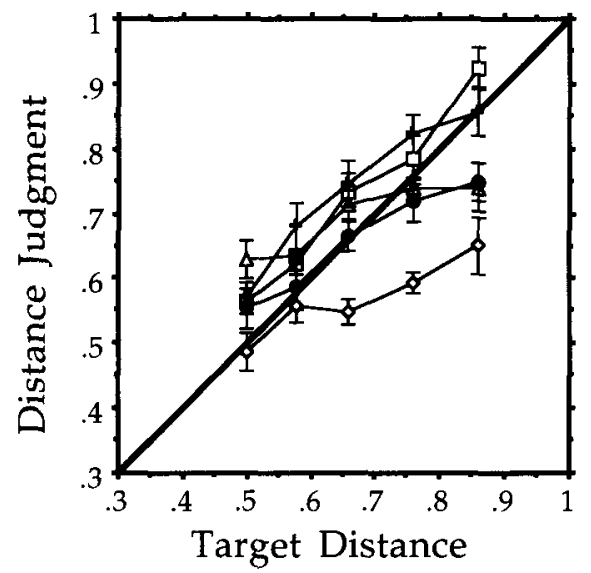

\section{Forward Without} Feedback

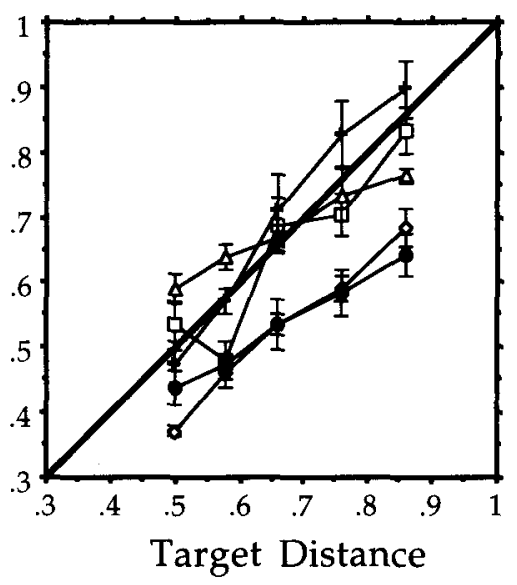

Side to Side Without Feedback

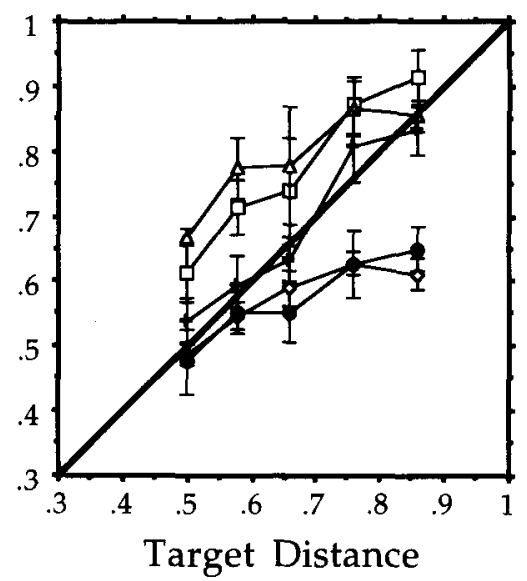

Figure 1. Mean reach distances (and standard error bars) for each observer in each head movement and feedback condition as a function of actual target distances. The distance judgments are in units proportional to the maximum reach distances for each observer. The closed circle and plus sign symbols represent the first and second authors (Observers 1 and 5) respectively, and the open triangle, square, and diamond symbols refer to naive Observers 2,3 and 4 , respectively. 
to-side condition had a mean slope of (.61), and the forward condition had a mean slope of (.75). Generally, in the two head movement conditions, the observers overestimated the distance of the near targets but underestimated the distance of the far targets. However, the over- and underestimations of distance in the side-to-side condition, accompanied by the higher intercept, yielded poorer overall accuracy than in the forward head movement condition. Further measures to test this accuracy will be discussed momentarily. These results indicate that, overall, the observers' distance judgments were better when using forward head movements than when using side-to-side head movements. The intercept difference for the feedback (.21) versus no-feedback (.17) conditions was significant (partial $F=4.5, p<.05$ ), and no significant difference in slopes was found. Finally, there was a significant interaction between the head movement and the feedback conditions (partial $F=5.4, p<.05$ ). Both head movement conditions yielded better performance with feedback than without, indicating that removal of feedback resulted in some loss of stability in reaching performance. Nevertheless, the forward head movement condition was better overall and had a smaller decrement in performance than did the side-to-side condition. This was revealed by simple regressions on each viewing and feedback condition (see Table 1 for mean slopes and reach values at distances .5 and .9 of maximum reach). ${ }^{2}$ The $r^{2}$ value decreased from .66 to .5 from the forward with feedback to the forward without feedback conditions. The $r^{2}$ values in the side-to-side conditions also decreased from .44 (side-toside with feedback) to .27 (side-to-side without feedback). The variance accounted for by the regressions in the sideto-side conditions was low.

We computed mean absolute errors and mean coefficients of variation (CVs) to compare accuracy and precision of reaches, respectively. Absolute errors for each observer, condition (head movement and feedback), and target distance were computed as the absolute difference of mean reach distance and target distance. We report mean absolute errors (with $S D$ s), in centimeters, as follows: forward with feedback, $2.6(2.1)$; side to side with feedback, 4.1 (3.1); forward without feedback, 5.1 (3.6); and side to side without feedback, 5.4 (4.1). A one-tailed paired $t$ test showed that the feedback conditions were significantly different in the forward head movement condition $[t(24)=$ $3.4, p<.01$, with a $2.5-\mathrm{cm}$ mean difference]. They were also significantly different in the side-to-side condition $[t(24)=1.7, p<.05$, with a $1.3-\mathrm{cm}$ mean difference $]$. Reaches performed with haptic feedback in both of the head movement conditions were more accurate than reaches performed without haptic feedback. We then compared the forward with feedback condition with the side to side with feedback condition and found a significant difference $[t(24)=2.4, p<.05]$, but no significant difference was found between the forward and the side-to-side conditions without feedback. This indicates that forward head movements allowed greater reduction of error with feedback than did the side-to-side head movements.

We also computed CVs (SDs of reaches/mean reach) for each observer and target distance in each of the four conditions. The mean CVs (with SDs) for each condition were as follows: forward with feedback, $8.6 \%(4.0 \%)$; side-to-side with feedback, $9.2 \%(2.9 \%)$; forward without feedback, $10.4 \%(4.2 \%)$; and side to side without feedback, $13.3 \%(5.7 \%)$. A comparison of individual conditions with $t$ tests revealed a significant difference between the forward and the side-to-side conditions without feedback, where the forward condition had better precision $[t(24)=2.4, p<.05]$. There was not, however, a significant difference between the forward and the lateral head movement conditions in the presence of feedback, indicating that reaches were equally precise for the two types of head movement when feedback was given $[t(24)=$ $0.46, p>.05]$. We also computed $t$ tests comparing feedback conditions and found that the forward with feedback condition was more precise than the forward without feedback condition $[t(24)=1.9, p<.05]$ and that the sideto-side with feedback condition was more precise than the side-to-side without feedback condition $[t(24)=3.2, p<$ $.01]$.

In addition to analyses on the reaches performed in the task, we also examined the kinematics of the observers' head movements. We analyzed the first five and the last five trials for each observer in the forward and side-to-side without feedback conditions. Table 2 shows the mean head amplitudes (with SDs) in centimeters and the periods of movement in seconds for each individual observer in both of the head movement conditions. We conducted a $2 \times$ $2 \times 5$ repeated measures analysis of variance (ANOVA) on amplitudes, comparing head movement conditions (forward or side to side), era (beginning or end of session), and trial (five at beginning and end of session). The only significant result was a main effect for direction of head movement. The forward head movements had signifi-

Table 1

Mean Slope, Standard Deviation, and Mean Reach Distances (as a

Proportion of Maximum Reach) at Target Distances .5 and .9 of Maximum Reach for Each Viewing Condition in Experiment 1

\begin{tabular}{lccccc}
\hline & & & \multicolumn{2}{c}{ Mean Reach Judgments } \\
\cline { 2 - 3 } \cline { 5 - 6 } \multicolumn{1}{c}{ Viewing Condition } & $M$ & $S D$ & & $\begin{array}{c}\text { Target at .5 of } \\
\text { Maximum Reach }\end{array}$ & $\begin{array}{c}\text { Target at .9 of } \\
\text { Maximum Reach }\end{array}$ \\
\hline Forward with feedback & .70 & .24 & .53 & .81 \\
Side to side with feedback & .61 & .25 & .56 & .81 \\
Forward with no feedback & .80 & .28 & .48 & .80 \\
Side to side with no feedback & .62 & .23 & .57 & .82 \\
\hline
\end{tabular}


Table 2

Mean and Standard Deviation Head Movement Amplitudes and Periods in Centimeters and Seconds, Respectively, for Each Observer in the Forward and Side-to-Side Without Feedback Conditions

\begin{tabular}{clccccc}
\hline & & \multicolumn{2}{c}{ Amplitude } & & \multicolumn{2}{c}{ Period } \\
\cline { 3 - 4 } \cline { 6 - 7 } Observer & Condition & $M$ & $S D$ & & $M$ & $S D$ \\
\hline \multirow{2}{*}{1} & Forward & 27.7 & 2.4 & & 2.3 & 0.08 \\
& Side to Side & 31.0 & 2.0 & & 3.0 & 0.10 \\
2 & Forward & 14.3 & 2.2 & & 2.2 & 0.16 \\
& Side to Side & 23.4 & 1.9 & & 2.4 & 0.11 \\
3 & Forward & 12.7 & 1.5 & & 1.9 & 0.13 \\
& Side to Side & 27.8 & 2.9 & 2.2 & 0.08 \\
4 & Forward & 21.5 & 4.5 & & 2.6 & 0.26 \\
\multirow{5}{*}{5} & Side to Side & 49.0 & 2.4 & 3.4 & 0.14 \\
& Forward & 16.5 & 1.8 & & 1.5 & 0.08 \\
Overall & Side to Side & 32.2 & 2.0 & & 1.9 & 0.07 \\
& Forward & 18.5 & 6.1 & & 2.1 & 0.40 \\
& Side to Side & 32.7 & 9.7 & 2.6 & 0.60 \\
\hline
\end{tabular}

cantly smaller amplitudes (mean $=18.5 \mathrm{~cm}, S D=6.1$ ) than did the side-to-side head movements [mean $=32.7 \mathrm{~cm}$, $S D=9.1 ; F(1,4)=12.4, p<.05]$. We also conducted a $2 \times$ $2 \times 5$ repeated measures ANOVA on the periods of head movement, using the same factors. Again, the only significant result was for direction of head movement. The forward head movements had significantly shorter periods (mean $=2.1 \mathrm{sec}, S D=0.4$ ) than did the side-to-side head movements [mean $=2.6 \mathrm{sec}, S D=0.6 ; F(1,4)=102.9, p<$ $.001]$.

\section{Discussion}

These results indicate that for monocular depth perception in a reaching task, forward head movements are more accurate than side-to-side head movements. Furthermore, when haptic feedback was removed, reaches in both of the head movement conditions became less accurate and less precise, but less so when using forward head movements than when using side-to-side head movements. The condition with the poorest performance in all respects was the side-to-side without feedback condition. The best performance was observed in the forward with feedback condition. The mean slope $(.70)$ was comparable with that found by Bingham and Pagano (1998) for forward head movements with the headcam and haptic feedback. As is shown in Table 1, the low slope reflected underestimation of distances. This is also consistent with the Bingham and Pagano finding.

Bingham and Pagano (1998) found comparable performances in terms of accuracy and slope with either headcam viewing or normal (unmediated) monocular viewing, although the level of precision was poorer with the headcam. In that study, both conditions were performed with haptic feedback.

Since the observers controlled their own head movements in each condition, it is possible that any differences in performance found between the forward and the sideto-side head movement conditions could be a function of the differences between the head movements. The results of the head motion analysis revealed that head movement amplitudes were significantly smaller in the forward head movement condition than in the side-to-side head movement condition. This shows that the superior performance in the forward head movement condition cannot be attributed to larger head movements. This difference in amplitude supports our inference that forward head movements yield better distance estimates. Smaller movements yield more accurate reaching. A difference in the period of head movements between the head movement conditions was also found. The forward head movements were found to take less time than the side-to-side head movements. This would be expected, given the smaller amplitudes of movement.

We next investigated reaching and normal unmediated monocular distance perception without haptic feedback in order to compare performances with both forward and side-to-side head movements and to determine whether the level of imprecision found in Experiment 1 without feedback was specific to viewing through the headcam.

\section{EXPERIMENT 2}

In the previous experiment, the observers wore the headcam in order to isolate the optic flow generated by their head movements. Although the headcam was required to ensure that optic flow was the only source of distance information, it was not representative of normal monocular viewing. The camera restricted the field of view to approximately $45^{\circ}$ and eliminated accommodation as well as vision of stable surface texture (Bingham \& Pagano, 1998). Bingham and Pagano found that performance with the headcam was more variable than that without the headcam, even with haptic feedback. In Experiment 2 , we compared reaching performances with forward versus side-to-side head movements. Normal unmediated monocular vision without haptic feedback was used to determine whether the level of precision would remain as poor as that found in Experiment 1 and whether, accordingly, the differences in the level of accuracy would be the same.

\section{Method}

Participants. Five people participated in this study. Three of the observers had also participated in Experiment 1 (Observers 1, 4, and 5), including the first two authors and one graduate student naive as to the purposes of the study. The remaining 2 observers were students, who were also naive as to the purpose of the study. All three students were paid $\$ 5$ an hour.

Procedure. Reaching performance in the forward and side-toside head movement conditions was tested with monocular viewing. Unlike the patch light conditions that obtained during the first experiment, normal lighting conditions were used, so that the observers could clearly see the entire target apparatus. Thus, all of the monocular cues to distance that are present in normal monocular vision were available for use in this condition: accommodation, surface texture, occlusion, and so forth. No feedback was given after a reach was performed. The observer's task was identical to that of Experiment 1, with one difference. In Experiment 1, the headcam display was blacked out (eliminating vision) once the observer initiated the reach. In the monocular condition used in Experiment 2, the observer closed his/her eye before starting the reach. Both conditions consisted of 25 experimental trials ( 5 target distances $\times 5$ target 
sizes), preceded by 12 practice trials. Therefore, the total number of trials run across both conditions was 74 .

\section{Results}

The results for each observer in each head movement condition are shown in Figure 2, where mean reach distances (and standard error bars) are plotted against target distances. We performed a multiple regression, as in Experiment 1 , to compare the performance in the forward head movement condition with that in the side-to-side head movement condition. The regression was significant $\left[r^{2}=\right.$ $.70, F(3,244)=187.5, p<.001]$. There was a significant difference in the intercept (partial $F=14.92, p<.001$ ), and the slope difference was not significant. The mean slope was .85 , and the separation between the two curves was $2.2 \mathrm{~cm}$. Separate simple regressions in each condition yielded an $r^{2}$ of .70 and a slope of .89 in the forward head movement condition and an $r^{2}$ of .69 and a slope of .81 in the side-to-side head movement condition. This was revealed by simple regressions on each viewing and feedback condition (see Table 3 for mean slopes and reach values at distances .5 and .9 of maximum reach).

As in Experiment 1, we calculated both the mean absolute error and the mean $\mathrm{CV}$ for both conditions. The mean absolute errors (with $S D$ s), in centimeters, were 4.2 (2.7) for the forward condition and 3.6 (2.9) for the side-to-side condition. No significant difference was found between the mean error values, indicating that performances in the two conditions were equally accurate $[t(24)=0.94, p>$ 05]. The mean CVs (with $S D$ s) were $3.7 \%(1.6 \%)$ for the forward monocular condition and $4.3 \%(2.6 \%)$ for the side-to-side monocular condition. A paired one-tailed $t$ test yielded no significant difference between the two con- ditions $[t(24)=1.12, p>.05]$. Performances were equally precise in the two conditions.

We also performed comparisons between the normal monocular conditions of Experiment 2 and the headcam monocular without feedback conditions of Experiment 1 (since no feedback was given during Experiment 2). First, we conducted a multiple regression to compare performance in the forward normal monocular condition with that in the forward headcam condition. The multiple regression was significant $\left[r^{2}=.31, F(3,240)=36.6, p<\right.$ $.001]$. There was a significant difference in the slopes of the two lines (partial $F=90.9, p<.001$ ). Separate simple regressions had yielded a slope of .89 for the normal monocular data and a slope of .80 for the headcam data. As would be expected, performance in the normal monocular condition was better than that in the headcam condition. We conducted a similar multiple regression comparing the side-to-side normal monocular condition with the side-to-side headcam condition. The regression was also significant $\left[r^{2}=.17, F(3,244)=16.3, p<.001\right]$, although the $r^{2}$ was lower. There was a significant slope difference between the side-to-side conditions (partial $F=35.6, p<$ .001 ). Simple regressions had yielded a slope of .81 for the side-to-side normal condition and a slope of .62 for the headcam condition. Performance was considerably better in the side-to-side normal monocular condition than in the side-to-side headcam condition.

We compared the mean CVs and absolute errors for each condition. The mean CVs (with $S D$ ) for each condition were as follows: forward monocular, $3.7 \%(1.6 \%)$; forward headcam, $10.4 \%(4.2 \%)$; side-to-side monocular, $4.3 \%(2.6 \%)$; side-to-side headcam, $13.3 \%(5.7 \%)$. Onetailed $t$ tests revealed significant differences in the CVs

\section{Forward Monocular}

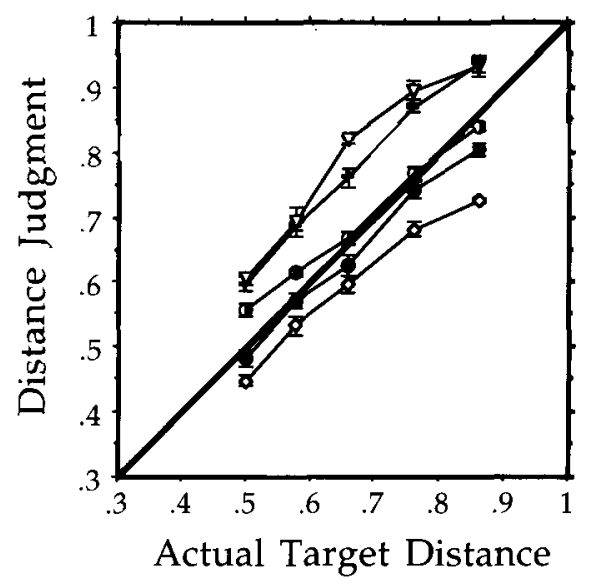

Side-to-Side Monocular

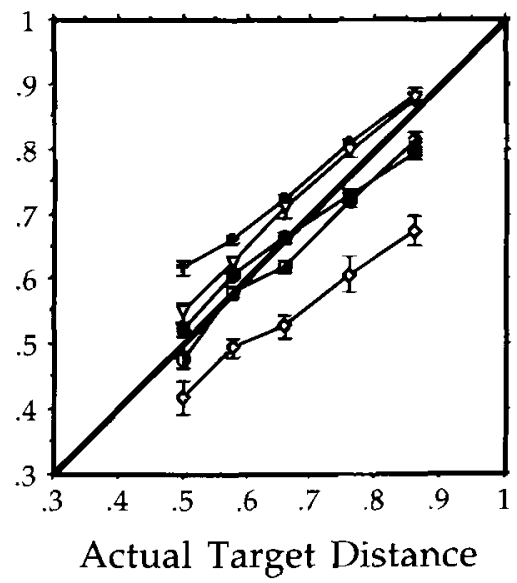

Figure 2. Mean reach distances (and standard error bars) for each observer in both of the normal monocular conditions as a function of actual target distances. The distance judgments are in units proportional to the maximum reach distances for each observer in Experiment 2 . The 3 observers from Experiment 1 are represented by the same symbols as in Figure 1 : closed circle and plus sign for the first and second authors (Observers 1 and 5) and open diamond for 1 naive observer (Observer 4 ), respectively. The upside-down triangle and the half-filled circle represent the $\mathbf{2}$ naive observers, who participated only in Experiment 2. 
Table 3

Mean Slope, Standard Deviation, and Mean Reach Distances (as a

Proportion of Maximum Reach) at Distances .5 and .9 of Maximum Reach for Each Viewing Condition in Experiment 2

\begin{tabular}{lccccc}
\hline & \multicolumn{2}{c}{ Slope } & & \multicolumn{2}{c}{ Mean Reach Judgments } \\
\cline { 2 - 3 } \cline { 5 - 6 } Viewing Condition & $M$ & $S D$ & & $\begin{array}{c}\text { Target at .5 of } \\
\text { Maximum Reach }\end{array}$ & $\begin{array}{c}\text { Target at .9 of } \\
\text { Maximum Reach }\end{array}$ \\
\hline Forward monocular & .89 & .08 & & .54 & .90 \\
Side-to-side monocular & .81 & .10 & & .52 & .84 \\
\hline
\end{tabular}

between the two forward conditions $[t(48)=7.5, p<.001]$, and between the two side-to-side conditions $[t(48)=7.2$, $p<.001]$. Performance in both the monocular conditions had much greater precision than did performance in both the headcam conditions. The mean absolute errors (with $S D$ s) for each condition, in centimeters, were as follows: forward monocular, 4.2 (2.7); forward headcam, 5.2 (3.6); side-to-side monocular, 3.6 (2.9); side-to-side headcam, 5.4 (4.1). A pairwise $t$ test revealed a significant difference between the two side-to-side head movement conditions $[t(48)=1.8, p<.05]$, where the side-to-side monocular condition had better accuracy. There was no significant difference in accuracy, however, between the forward monocular and the forward headcam conditions.

\section{Discussion}

Overall, the results of Experiment 2 indicate that in normal monocular conditions, performance when using sideto-side head movements is as accurate and precise as when using forward head movements. Performance in the headcam conditions of Experiment 1 was less accurate in the side-to-side head movement conditions and more variable overall. The presence of additional monocular visual information, such as accommodation, surface texture, dynamic occlusion, and size, in Experiment 2 improved performance. The poorer performance in the headcam conditions could be due to the additional weight added by the headcam apparatus, which could cause minor perturbations in kinesthetic information, as well as in basic movements. However, we would predict the additional weight would yield a consistent trend of either over- or underestimation in reaches. Neither particular trend was observed, however. We found mostly individual differences, a trend that Bingham, Zaal, Robin, \& Shull (in press) observed in a condition without the headcam. Therefore, we do not attribute the increase in performance seen in the normal monocular condition to the removal of the weight added by the headcam apparatus but, rather, to the presence of additional monocular information.

\section{GENERAL DISCUSSION}

The first purpose of the experiments described here was to investigate which type of head movement yielded better distance judgments in a monocular reaching task. The results of the experiments indicated that performance was both more accurate and more precise when using forward head movements to generate optic flow information in the absence of other visual information. The mean absolute error values and mean CVs indicated that the observers were more accurate and more precise in their reaches. On the other hand, Marotta, Perrot, Nicolle, et. al. (1995) found that monocular people tended, over time, to prefer to rotate the head sideways to obtain depth information from motion parallax and stopped using forward head movements. They argued that recently enucleated patients should be trained to use these types of head movements, on the basis of the assumption that the people switched to this type of head movement because the information they obtained from motion parallax was more informative to them. In Experiment 1, we isolated these types of optic flow and found that reaching performance was actually worse in the side-to-side conditions that generated motion parallax than in the forward conditions that generated radial flow patterns in addition to some parallax. The presence of both types of information when the forward head movement was employed could allow for more accurate perception of distance. The side-to-side head movements yielded a poorer performance, even though observers used significantly larger head movements and took more time than in the forward direction.

The second purpose of these experiments was to investigate how stable the observers' reaching performance was after the removal of haptic feedback. The headcam apparatus was used to isolate optic flow as information, but it introduced a large perturbation to vision. It eliminated all other possible sources of information, such as ocular parallax (Bingham, 1993a), convergence, binocular disparity, and accommodation. Bingham and Pagano (1998) used the headcam to investigate performance in a similar reaching task with haptic feedback, but they did not address the stability of performance after the removal of feedback. Our experiment showed that for both of the head movement conditions, accuracy and precision deteriorated when feedback was removed. The deterioration in performance was greater, however, for the side-to-side head movement condition, indicating greater instability than with forward head movements.

Nevertheless, when performance was compared under normal unmediated monocular viewing conditions, performance with side-to-side head movements was virtually equal to that with forward head movements. This implies that the parallax can be used equally well to scale other stable sources of information about relative distance, such as visible surface texture, so that they might act as information about definite distance. Accordingly, we suggest 
that the enucleated patients in Marotta, Perrot, Nicolle, \& Goodale's (1995) study did not learn to perform sideways movements because the information from motion parallax was more useful, but because the action itself was more efficient and easier to perform, while allowing equal performance. However, sideways movements may not be more efficient in other situations. For example, when reaching for an object just out of arm's reach, people naturally produce forward head movement. It would be unnatural and inconvenient to employ a side-to-side type of head movement in this situation. Also, forward head movement accompanies locomotor movements in walking or running, where scaling information could be provided in terms of stride length. Both of these examples indicate that people might be more adept or practiced at using egocentric distance information generated by forward head movements.

\section{REFERENCES}

Bingham, G. P. (1993a). Optical flow from eye movement with head immobilized: "Ocular occlusion" beyond the nose. Vision Research, 33, 777-789.

Bingham, G. P. (1993b). Perceiving the size of trees: Biological form and the horizon ratio. Perception \& Psychophysics, 54, 485-495.

Bingham, G. P., \& Pagano, C. C. (1998). The necessity of a perception/action approach to definite distance perception: Monocular distance perception for reaching. Journal of Experimental Psychology: Human Perception \& Performance, 24, 1-25.

BinghaM, G. P., \& STASSEN, M. G. (1994). Monocular distance information in optic flow from head movement. Ecological Psychology, 6, 219-238.

BinghaM, G.P. \& ZAAL, F. (1997a, November). Haptic feedback is required for accurate and stable distance and shape perception. Paper presented at the 38th Annual Meeting of the Psychonomic Society, Philadelphia.

Bingham, G. P., \& ZaAL, F. (1997b, October). The importance of haptic feedback for the stability of distance and shape perception used to guide reaching. Paper presented at the Minnesota Conference on $\mathrm{Vi}$ sion for Reaching and Grasping, University of Minnesota, Minneapolis.

Bingham, G. P., ZaAl, F., Robin, D., \& Shull, J. A. (in press). Distances in definite distance and shape perception as measured by reaching with and without haptic feedback. Journal of Experimental Psychology: Human Perception \& Performance.

Ellard, C. G., Goodale, M. A., \& Timney, B. (1984). Distance estimation in the Mongolian gerbil: The role of dynamic depth cues. Behavioural Brain Research, 14, 29-39.

ERIKSSON, E. S. (1974). Motion parallax during locomotion. Perception \& Psychophysics, 16, 197-200.

FERRIS, S. H. (1972). Motion parallax and absolute distance. Journal of Experimental Psychology, 95, 258-263.

Foley, J. M. (1977). Effect of distance information and range on two indices of visually perceived distance. Perception, 6, 449-460.

Foley, J. M. (1978). Primary distance perception. In R. Held, H. W. Lei- bowitz, \& H. L. Teuber (Eds.), Handbook of sensory physiology: Vol. 8. Perception (pp. 181-213). Berlin: Springer-Verlag.

FOLEY, J. M., \& HELD, R. (1972). Visually directed pointing as a function of target distance, direction, and available cues. Perception \& Psychophysics, 12, 263-268.

Gogel, W. C., \& Tietz, J. D. (1973). Absolute motion parallax and the specific distance tendency. Perception \& Psychophysics, 13, 284-292.

GoGEL, W. C., \& TieTZ, J. D. (1979). A comparison of oculo-motor and motion parallax cues of egocentric distance. Vision Research, 19, 1161-1179.

JohansSON, G. (1973). Monocular movement parallax and near space perception. Perception, 2, 135-146.

Marotta, J. J., Perrot, T. S., Nicolle, D., \& Goodale, M. A. (1995). The development of adaptive head movements following enucleation. Eye, 9, 333-336.

Marotta, J. J., Perrot, T. S., Nicolle, D., Servos, P., \& Goodale, M. A. (1995). Adapting to monocular vision: Grasping with one eye. Experimental Brain Research, 104, 107-114.

Pagano, C. C., \& Bingham, G. P. (1998). Comparing measures of monocular distance perception: Verbal and reaching errors are not correlated. Journal of Experimental Psychology: Human Perception \& Performance, 24, 1037-1051.

PEDHAZUR, E. (1982). Multiple regression in behavioral analysis. New York: Holt, Rinehart \& Winston.

Rogers, B. J. (1993). Motion parallax and other dynamic cues for depth in humans. In R. A. Miles \& J. Wallman (Eds.), Visual motion and its role in the stabilization of gaze (pp. 119-137). Amsterdam: Elsevier.

Rogers, S., \& Rogers, B. J. (1992). Visual and nonvisual information disambiguate surfaces specified by motion parallax. Perception \& Psychophysics, 52, 446-452.

Runeson, S., \& FryKHOLM, G. (1981). Visual perception of lifted weight. Journal of Experimental Psychology: Human Perception \& Performance, 7, 733-740.

Servos, P., Goodale, M. A., \& Jakobson, L. S. (1992). The role of binocular vision in prehension: A kinematic analysis. Vision Research, 32, 1513-1521.

\section{NOTES}

1. We refer to definite as opposed to absolute distance, to acknowledge the noise inherent to actual measurements. The term absolute implies absolute precision and accuracy. This issue is discussed at length in Bingham (1993b) and Bingham and Pagano (1998). Also note that, dimensionally, optical patterns are angular (and temporal), so lengths are not preserved when mapped onto optical patterns, as is demonstrated by the inability to describe optical pattern in terms of meters, inches, or any metric length unit.

2. Note that the slope alone does not determine accuracy of performance. The placement of the curve is also important. It is possible to observe better accuracy for some curves with lower slopes than for curves for higher slopes (see forward with feedback vs. forward no feedback). The slope is used in specific cases to describe the trend that produced inaccuracy. Low slope, for instance, could reflect either overestimation of near targets and underestimation of far targets or progressively greater underestimation of increasingly far targets. The former trend would yield greater overall accuracy.

(Manuscript received July 21, 1998 ; revision accepted for publication August 9, 1999.) 